\title{
Como se enxergam treinadores e praticantes de natação?
}

How do coaches and swimmers see themselves?

Milena Schiezari Ru Barnabe ${ }^{1}$, Hélio Mamoru Yoshida ${ }^{1}$, João Guilherme Cren Chiminazzo², Vinicius Nagy Soares ${ }^{1}$, Paula Teixeira Fernandes ${ }^{1}$

${ }^{1}$ Universidade Estadual de Campinas (Unicamp), Campinas/SP, Brasil

${ }^{2}$ Centro Universitário de Jaguariúna (UniFAJ), Jaguariúna/SP, Brasil

\section{HISTÓRICO DO ARTIGO}

Recebido: 01 setembro 2019 Revisado: 06 abril 2020

Aprovado: 09 abril 2020

\section{PALAVRAS-CHAVE:}

Psicologia do Esporte; Relação Treinador-Atleta; Comportamento.

\section{KEYWORDS:}

Sport Psychology; Coach-Athlete Relationship; Behavior.

\section{RESUMO}

INTRODUÇÃO: A prática esportiva, especificamente a natação, está entre as principais modalidades praticadas por crianças e adolescentes no Brasil.

OBJETIVO: Descrever condutas de treinadores de natação e verificar como essas condutas são percebidas pelos atletas.

MÉTODOS: Foram entrevistados 30 nadadores $(15,31 \pm 2,47$ anos) e 5 treinadores, através da Escala de Comportamento do Treinador - Versão Atleta e da Escala de Comportamento de Treinadores Desportivos Versão Treinadores.

RESULTADOS: Foi identificada correspondência entre as opiniões, demonstrando que as percepções dos treinadores são similares às percepções dos atletas de natação.

CONCLUSÃO: Os treinadores avaliados apresentaram preocupação com a formação abrangente dos praticantes, sobretudo em relação aos aspectos técnicos, físicos e psicológicos.

\section{ABSTRACT}

BACKGROUND: Sport, specifically swimming, is among the main sports practiced by children and adolescents in Brazil.

OBJECTIVE: To describe the conduct of swimming coaches and to verify how these behaviors are perceived by athletes.

METHODS: 30 swimmers (15.31 \pm 2.47 years) and 5 coaches were interviewed through the Coach Behavior Scale - Athlete Version and the Sports Coach Behavior Scale - Coach Version.

RESULTS: We identified a correspondence between opinions, demonstrating that coaches 'perceptions are similar to swimming athletes' perceptions.

CONCLUSION: The investigated coaches were concerned with the comprehensive training of practitioners, especially regarding the technical, physical and psychological aspects. 


\section{INTRODUÇÃO}

A natação é uma das modalidades esportivas mais praticadas no Brasil, tendo a maior concentração de praticantes na adolescência (BRASIL, 2015). A natação pode ser definida como a habilidade de se movimentar no meio líquido, o que é possível graças às forças propulsivas, decorrentes da ação dos membros superiores e inferiores, vencendo a resistência exercida pela água (SAAVEDRA; ESCALANTE; RODRÍGUEZ, 2003).

A prática regular pode fornecer diferentes benefícios à saúde, como a melhora da função respiratória (TAHARA; SANTIAGO; TAHARA, 2006), diminui a incidência de doenças crônicas, como diabetes e hipertensão (CHASE; SUI; BLAIR, 2008), aumento da força e da resistência muscular (TEIXEIRA, 2008), além de elevar a autoestima (TAHARA; SANTIAGO; TAHARA, 2006) e a autoconfiança dos seus praticantes (TEIXEIRA, 2008). Ademais, diminui o estresse (BERGER; OWEN, 1988), depressão (CID; SILVA; ALVES, 2007), e melhora a imagem corporal (CID; SILVA; ALVES, 2007).

Assim, os benefícios da prática da natação supracitados podem ser potencializados a partir da relação entre o treinador/professor e o atleta/aluno, transferindo, desta forma, os ganhos da prática para o dia-dia do aluno (GOULD; CARSON, 2010). Portanto, a relação dos praticantes, especificamente dos atletas e treinadores é um tema bastante explorada em outras modalidades pela Psicologia do Esporte (GOMES; PAIVA, 2010; LEITÃO, 1999), a fim de motivá-los a alcançarem seus objetivos, levando ao desenvolvimento saudável e duradouro na modalidade. Essa temática se caracteriza como relação treinador-atleta. Assim, as atitudes e os valores dos treinadores influenciam os atletas na continuidade da modalidade esportiva, sendo a comunicação um elemento-chave para o bom relacionamento interpessoal (SMITH; SMOLL; CUMMING, 2007).

Diferentes estudos abordaram como o treinador pode influenciar nos resultados, tanto em competição quanto em treinamentos (ALBUQUERQUE MORAES et al., 2010; BRANDÃO; CARCHAN, 2010; CRUZ et al., 2001; LÔBO; ALBUQUERQUE MORAES; NASCIMENTO, 2005). Nesse sentido, o treinador deve se adaptar de acordo com as situações do cotidiano, o que torna essa "volatilidade" um elemento importante para a resolução de conflitos circunstanciais (ZHANG; JENSEN; MANN, 1997).

Outro ponto a ser considerado é a diferença da percepção de liderança entre os membros da equipe (SIMÕES, 1987), pois cada atleta interpreta de forma pessoal as atitudes do treinador, podendo a comunicação aumentar a autoestima e despertar o interesse pela modalidade (BRANDÃO; CARCHAN, 2010). Assim, o treinador pode ser um referencial na orientação, identificação e delimitação dos objetivos a serem cumpridos ao longo da carreira, mantendo o praticante otimista e confiante para alcançar suas metas e, consequentemente, obter sucesso na modalidade (SILVEIRA, 2005).

Dessa forma, Chelladurai e Saleh, (1980) aperfeiçoou a Leadership Scale for Sports (LSS) realizada no ano de 1978, classificando as condutas mais frequentes dos treinadores em: a) Treinamento Instrução, que objetiva auxiliar tecnicamente e taticamente sobre a modalidade esportiva, estruturando e coordenando de maneira satisfatória as atividades dos atletas; $b$ ) Comportamento Democrático, que proporciona um ambiente democrático, tornando os integrantes da equipe participantes diretos na preparação global e específica dos treinamentos, permitindo maior interação nas decisões relacionadas ao grupo, mas a decisão final ainda pertence ao treinador; c) Comportamento Autocrático, no qual disciplina e eficiência são cobradas enfaticamente, com ausência de qualquer interação nas decisões; d) Suporte Social, que leva em consideração não somente se o grupo evolui seu desempenho, mas também se cada participante está satisfeito e se a relação entre os mesmos acontece de maneira tranquila e afetuosa; e) Feedback Positivo, no qual podem ser concedidos elogios, valores em dinheiro e até mesmo objetos simbólicos (medalhas, placas de agradecimento), retribuindo aos feitos conquistados e motivando os praticantes a buscarem novos objetivos.

Ademais, Zhang, Jensen e Mann (1997) acrescentam mais uma conduta além das supracitadas, a consideração da situação, que é a conduta que o treinador se apropria após considerar os fatores da situação na qual abrange o tempo, o indivíduo, o ambiente e o jogo. Assim, os treinadores podem possuir certos comportamentos positivos durante os treinamentos como, disponibilizar o cronograma de treinamentos e se preocupar com a técnica específica de seus atletas para com a modalidade; permitir a livre expressão de seus atletas e ouvi-los; proporcionar um ambiente harmônico para que o atleta sinta confiança em expressar situações de dentro e de fora do meio esportivo ou, contrariamente, desconsiderar a opinião e gritar com os atletas caracteriza um comportamento negativo (LÔBO; ALBUQUERQUE MORAES; NASCIMENTO, 2005). A predominância de algum desses comportamentos utilizados pelos treinadores, caracteriza seu estilo de liderança na equipe.

Além disso, vale ressaltar que as relações treinador-atleta devem ser imbuídas de respeito e confiança, sendo que os treinadores deveriam ser os protagonistas desta realidade, propondo oportunidades positivas para seu desenvolvimento. Assim, este estudo buscou detalhar as condutas dos treinadores de uma modalidade olímpica individual, a natação, que podem estar presentes no dia a dia dos treinamentos podendo auxiliar os profissionais dessa área esportiva, visto que na literatura ainda pouco se encontra sobre essa temática, especificamente na natação.

Dessa forma, este estudo teve por objetivo descrever as condutas dos treinadores de natação e verificar como essas condutas são percebidas pelos seus atletas.

\section{MÉODOS}

Participaram do estudo 30 atletas de natação (18 homens), com média de idade de 15 anos $( \pm 2,47)$, experiência de 5 anos $( \pm 2,8)$ na modalidade, sendo que 14 voluntários estavam na equipe desde o ingresso na natação. Cinco treinadores foram entrevistados, todos do sexo masculino, com média de idade de 37 anos $( \pm 10,9)$, experiência de 15 anos $( \pm 10,7)$ na modalidade, sendo 10 anos $( \pm 8,1)$ como treinadores. Foi considerado como critério de exclusão, participantes com déficit cognitivo que afetou a compreensão dos instrumentos utilizados nessa pesquisa. A pesquisa foi realizada em cinco clubes de natação do estado de São Paulo. O estudo foi aprovado pelo Comitê de Ética em Pesquisa da UNICAMP 
(CAAE N. 39742514.8.0000.5404, Parecer N. 938.572).

Foram utilizados quatro instrumentos: Ficha de identificação do treinador; Ficha de identificação do atleta; Escala de Comportamento do Treinador (ECT-A); e Escala de Comportamento de Treinadores Esportivos (ECT-T):

Ficha de identificação para treinadores: 13 perguntas de caráter pessoal (sexo, escolaridade, doenças, formação e complementação acadêmica, etc.), dados sobre a experiência de atividade física (tempo de atuação em esporte organizado, na modalidade atual e na equipe em questão) e uma percepção sobre sua relação com os atletas (nota de 0 a 10).

Ficha de identificação para atletas: 18 perguntas de caráter pessoal (sexo, idade, escolaridade, peso, altura, entre outras) dados sobre a experiência em atividade física (quanto tempo está envolvido em práticas esportivas, na modalidade, tempo com o treinador atual, tempo de dedicação ao treinamento etc.), além de uma percepção sobre sua relação com o treinador (nota de 0 a 10).

Escala de Comportamento de Treinadores Esportivos (ECT-T) - Versão treinadores: traduzido e validado no Brasil: verifica se o treinador detém boa percepção sobre o seu próprio comportamento em relação aos atletas (SILVEIRA, 2005). As questões são do tipo Likert, variando de 1 (nunca) a 5 (sempre).

Escala de Comportamento do Treinador (ECT-A) - Versão Atleta: traduzido e validado no Brasil: tem como objetivo verificar a percepção do atleta sobre o comportamento do treinador (LÔBO; ALBUQUERQUE MORAES; NASCIMENTO, 2005). Para todas as questões, utiliza-se escala no qual 1 representa "nunca" e 7 que representa "sempre" referente a frequência dos comportamentos específicos de seu treinador. No final de cada dimensão, contém espaço destinado a acrescentar comentários referentes à percepção do comportamento do treinador, de acordo com as questões tratadas.

Ambos os instrumentos ECT-T e ECT-A foram validados par ao Brasil. O ECT-A foi resultado da tradução realizada por Lôbo, Albuquerque Moraes e Nascimento (2005) do Coaching Behavior Scale for Sport (CBS-S) versão proposta por Côté et al. (1999). Ambos os questionários abrangem seis dimensões do comportamento do treinador:

1. Treinamento e Condicionamento Físico (disponibilidade do planejamento de treinamento pelos treinadores e a confiança dos atletas a ele, 7 questões)

2. Treinamento Técnico (o treinador demonstra atenção e atitude na correção de erros técnicos específicos da modalidade por diferentes formas, além de se certificar que o atleta entendeu corretamente, 8 questões);

3. Preparação Mental (o treinador empenha-se em aconseIhar seus atletas a confiarem em si mesmos, manterem concentração nos seus objetivos e serem positivos em relação à prática esportiva, 5 questões);

4. Estabelecimento de Metas (os atletas percebem o quanto seus treinadores estão envolvidos e preocupados com seus objetivos a curto e a longo prazo, 6 questões);

5. Reforço Pessoal Positivo (confiança dos atletas nos treinadores quanto às relações fora do campo esportivo, mostrando-se sempre disponíveis e compreensíveis, 6 questões);

6. Reforço Pessoal Negativo (o treinador possui atitudes que desfavorecem o "bom convívio", como gritar, desconsiderar a opinião dos atletas, 8 questões).

Um avaliador foi encarregado de frequentar o local de treinamento e aplicar os questionários. Não houve prejuízo ao treinamento dos atletas, pois foi escolhido, junto ao treinador, o horário mais adequado para a aplicação dos instrumentos.
Foi entregue tanto aos treinadores quanto aos atletas, uma ficha de identificação para registro de dados pessoais, além de uma pergunta que solicitava aos voluntários que atribuíssem uma nota de 0 a 10 sobre a relação treinador-atleta. Foram aplicadas também a Escala de Comportamento do Treinador versão atleta (ECT- A).

O tempo aproximado para responder os questionários foi de 15 minutos, mantendo-se as mesmas condições de aplicação para todos os participantes. Para a análise dos resultados, foi adotado o método descritivo, sendo calculada a média, o desvio padrão e o percentual relativo de cada resposta dos questionários.

\section{RESULTADOS}

Os atletas do estudo estavam com seus treinadores há 2 anos $( \pm 1,33)$ e dedicavam em média 22 horas semanais nas rotinas de treinamento. Sobre as avaliações da relação treinador-atleta, em média, os atletas atribuíram a nota $8,81( \pm 1,11)$. A percepção dos treinadores quanto a relação treinador atleta foi de $8,33( \pm 1,53)$.

Na Tabela 1, estão descritas as questões respondidas pelos participantes do estudo. A partir da comparação dos valores percentuais, identificamos correspondência entre as opiniões, demonstrando que as percepções foram similares.

Sobre o treinamento físico, os atletas se mostraram mais otimistas do que os treinadores em relação aos programas de condicionamento físico. Sobre o treinamento técnico, os treinadores demonstraram empenho em orientar, corrigir e garantir a assimilação dos conteúdos, como indicam os exemplos: "Ele sempre fica em cima da gente no preparo e sempre está planejando os treinos"; "Ele sempre corrige nossos erros e sempre quer o nosso melhor"; "Meu técnico é muito atencioso com o quesito das técnicas, ele nos ajuda sempre que algo está errado".

Em relação ao treinamento mental, embora os treinadores possam ter subvalorizado suas próprias condutas, como em relação ao "aconselhamento", os atletas observaram no treinador uma liderança fundamental para habilidades emocionais como autoestima, autoconfiança e motivação. "Ele tenta nos deixar o mais confiante possível mentalmente"; "Nas competições ele dá todo apoio possível para nós nos concentrarmos na prova". O estabelecimento de metas foi avaliado positivamente pelos atletas, assim como a confiança deles em relação ao treinador. Condutas como "ignorar a opinião do atleta" ou "gritar", não foram expressivas.

É importante ressaltar que alguns estudos abordam o comportamento do treinador como o estilo de liderança que ele possui com os seus atletas, observando qual é o preferido por eles em diferentes faixas etárias no badminton (CRUZ; KIM, 2017), no triathlon (MATIAS et al., 2014) e em mais modalidades (HORN et al., 2011) utilizando a Leadership Scale for Sport (LSS) atualizada por Chelladurai (2007). Outros estudos utilizaram a Escala de Liderança no Desporto (ELD) no futsal e futebol (LESSA; MORAES; LIMA, 2013) e por fim, a Coaching Behavior Scale for Sport (CBS-S) na validação sueca.

Isto posto, temos que diante do objetivo de descrever as percepções dos atletas sobre as condutas dos treinadores, verificamos aproximação das opiniões entre as partes. Inferi- 
mos que os treinadores estão preocupados com uma formação abrangente de seus atletas, preocupando-se com o bom relacionamento, tanto nos aspectos relacionados às questões pessoais - emocionais e cognitivas - quanto nas questões relacionadas à modalidade - técnicas, táticas e carreira esportiva.

Tabela 1. Média, desvio padrão e percentual das questões extraídas dos questionários ECT-A e ECT-T.

\begin{tabular}{|c|c|c|}
\hline Perguntas & $\begin{array}{l}\text { Treinadores } \\
\text { Média } \pm D P \\
\text { (\% do máximo) }\end{array}$ & $\begin{array}{c}\text { Atletas } \\
\text { Média } \pm D P \\
\text { (\% do máximo) }\end{array}$ \\
\hline \multicolumn{3}{|l|}{ Treinamento físico } \\
\hline $\begin{array}{l}\text { Forneço um programa de condi- } \\
\text { cionamento físico no qual o atleta } \\
\text { confia. }\end{array}$ & $\begin{array}{c}4,20 \pm 0,75 \\
(84,00)\end{array}$ & $\begin{array}{c}6,67 \pm 0,56 \\
(95,29)\end{array}$ \\
\hline $\begin{array}{l}\text { Forneço um programa anual de } \\
\text { treinamento. }\end{array}$ & $\begin{array}{c}4,00 \pm 0,89 \\
(80,00)\end{array}$ & $\begin{array}{c}5,46 \pm 1,91 \\
(77,98)\end{array}$ \\
\hline \multicolumn{3}{|l|}{ Treinamento técnico } \\
\hline $\begin{array}{l}\text { Forneço ao atleta uma resposta (fe- } \\
\text { edback) para corrigir erros técnicos. }\end{array}$ & $\begin{array}{c}4,20 \pm 0,75 \\
(84,00)\end{array}$ & $\begin{array}{c}5,71 \pm 1,90 \\
(81,55)\end{array}$ \\
\hline $\begin{array}{l}\text { Forneço ao atleta orientações } \\
\text { (feedback) que o ajuda a melhorar } \\
\text { sua técnica. }\end{array}$ & $\begin{array}{l}4,40 \pm 0,80 \\
(88,00)\end{array}$ & $\begin{array}{l}6,00 \pm 1,35 \\
(85,71)\end{array}$ \\
\hline $\begin{array}{l}\text { Certifico-me de que o atleta esteja } \\
\text { entendendo as técnicas e as estraté- } \\
\text { gias que Ihe estão sendo ensinadas }\end{array}$ & $\begin{array}{c}4,00 \pm 0,00 \\
(80,00)\end{array}$ & $\begin{array}{c}6,04 \pm 1,23 \\
(86,31)\end{array}$ \\
\hline \multicolumn{3}{|l|}{ Treinamento mental } \\
\hline $\begin{array}{l}\text { Dou conselhos ao atleta sobre como } \\
\text { atuar sob pressão. }\end{array}$ & $\begin{array}{c}4,20 \pm 0,75 \\
(84,00)\end{array}$ & $\begin{array}{c}5,00 \pm 1,25 \\
(71,43)\end{array}$ \\
\hline $\begin{array}{l}\text { Dou conselhos ao atleta sobre como } \\
\text { permanecer confiante em suas } \\
\text { habilidades. }\end{array}$ & $\begin{array}{c}4,40 \pm 0,49 \\
(88,00)\end{array}$ & $\begin{array}{c}5,71 \pm 1,27 \\
(81,55)\end{array}$ \\
\hline $\begin{array}{l}\text { Dou conselhos ao atleta sobre como } \\
\text { ser positivo consigo mesmo. }\end{array}$ & $\begin{array}{c}4,00 \pm 0,63 \\
(80,00)\end{array}$ & $\begin{array}{c}5,83 \pm 1,46 \\
(83,33)\end{array}$ \\
\hline $\begin{array}{l}\text { Dou conselhos ao atleta sobre como } \\
\text { permanecer focalizado. }\end{array}$ & $\begin{array}{c}3,60 \pm 0,80 \\
(72,00)\end{array}$ & $\begin{array}{c}6,21 \pm 0,93 \\
(88,69)\end{array}$ \\
\hline \multicolumn{3}{|l|}{ Estabelecimento de metas } \\
\hline $\begin{array}{l}\text { Ajudo-o a estabelecer objetivos a } \\
\text { longo prazo. }\end{array}$ & $\begin{array}{c}4,40 \pm 0,80 \\
(88,00)\end{array}$ & $\begin{array}{c}6,00 \pm 1,47 \\
(85,71)\end{array}$ \\
\hline $\begin{array}{l}\text { Demonstro compromisso com os } \\
\text { objetivos do atleta. }\end{array}$ & $\begin{array}{c}4,20 \pm 0,98 \\
(84,00)\end{array}$ & $\begin{array}{c}6,77 \pm 0,44 \\
(96,70)\end{array}$ \\
\hline \multicolumn{3}{|l|}{ Enquanto treinador esportivo... } \\
\hline $\begin{array}{l}\text { Sou digno de confiança com relação } \\
\text { aos problemas pessoais do atleta. }\end{array}$ & $\begin{array}{l}3,80 \pm 0,40 \\
(76,00)\end{array}$ & $\begin{array}{c}6,23 \pm 0,93 \\
(89,01)\end{array}$ \\
\hline $\begin{array}{l}\text { Sou facilmente acessivel sobre os } \\
\text { problemas pessoais que o atleta } \\
\text { possa ter. }\end{array}$ & $\begin{array}{c}4,00 \pm 0,63 \\
(80,00)\end{array}$ & $\begin{array}{c}6,23 \pm 1,24 \\
(89,01)\end{array}$ \\
\hline $\begin{array}{l}\text { Grito com o atleta quando estou } \\
\text { com raiva. }\end{array}$ & $\begin{array}{c}2,80 \pm 0,98 \\
(56,00)\end{array}$ & $\begin{array}{c}3,69 \pm 2,29 \\
(52,75)\end{array}$ \\
\hline $\begin{array}{l}\text { Demonstro favoritismo por algum } \\
\text { atleta. }\end{array}$ & $\begin{array}{c}1,80 \pm 0,75 \\
(36,00)\end{array}$ & $\begin{array}{l}3,62 \pm 2,57 \\
(51,65)\end{array}$ \\
\hline Ignoro a opinião do atleta. & $\begin{array}{c}1,60 \pm 0,80 \\
(32,00)\end{array}$ & $\begin{array}{c}2,62 \pm 2,10 \\
(37,36)\end{array}$ \\
\hline
\end{tabular}

Os treinadores apresentaram condutas de treino-instrução por se preocuparem em passar um programa de treinamento voltado às características específicas da modalidade, no qual o aperfeiçoamento técnico e tático consiste em um objetivo do treinador, o que é percebido pelos praticantes. Isso demonstra atenção com o desempenho dos seus atletas, sobretudo em relação à aprendizagem da técnica. Além disso, os valores altos e próximos, de ambas as amostras revela que os atletas apresentaram percepção positiva sobre as condutas dos treinadores, o que segundo Serpa (2002), favorece a permanência na modalidade. Ademais, a boa relação entre treinadores e atletas proporciona experiências positivas que se referem às "habilidades pessoais e sociais, iniciativa e autoestima", que, somadas aos aspectos específicos da modalidade, permitem o desenvolvimento integral do atleta (VELLA; OADES; CROWE, 2013, p. 552). Esses resultados corroboram estudos anteriores (ARDUA; MÁRQUEZ, 2007; HOSHINO; SONOO; VIEIRA, 2007; SHERMAN; FULLER; SPEED, 2000; VILANI, 2004) em que os atletas também preferiram condutas de treino-instrução.

A preferência por esse tipo de conduta já foi evidenciada tanto em esportes coletivos quanto individuais (HOSHINO; SONOO; VIEIRA, 2007). E em estudo envolvendo atletas de alto nível do tênis de mesa, foi destacada a importância do reforço positivo (VILANI, 2004). Em equipes coletivas, foi observada a preferência pelo comportamento de treino-instrução e de feedback positivo, como apontam os estudos sobre nado sincronizado (ARDUA; MÁRQUEZ, 2007) e basquetebol (SHERMAN; FULLER; SPEED, 2000).

Albuquerque Moraes et al. (2010) não encontraram proximidade entre as respostas dos atletas e treinadores. Segundo os autores, isso ocorreu por conta da má organização dos treinamentos e de comunicação ineficiente entre os membros da equipe. Dessa forma, a boa relação do treinador com os atletas e a orientação adequada das características específicas da modalidade são essenciais para a satisfação com a prática esportiva, despertando o desejo de aprimoramento e de permanência dos praticantes (CATITA, 2002, p. 6) e, consequentemente, para o sucesso no esporte (CHEUCZUK et al., 2016).

Dentro dos resultados destacamos os aspectos do treinamento mental. Mostramos que os atletas percebem seus treinadores enfatizam a melhora do repertório no quesito "permanecer focalizado", com $88,69 \%$ do total, em contraste com $72 \%$ da própria percepção dos treinadores. Assim, apesar dos treinadores não se perceberem com atitudes voltadas à concentração e no foco, os atletas tiveram essa percepção.

Paralelamente a isso, os treinadores acreditam que suas condutas são para aconselhamentos, como por exemplo "permanecer confiante com suas habilidades", com $88 \%$. Este fato pode ser explicado Fortes et al. (2016), quando demostram que a melhora da confiança, após o treinamento mental, resulta na melhora do desempenho de nadadores. Assim, apesar dos atletas não perceberem as ações para a melhora da confiança nas habilidades, os treinadores tiveram esta conduta.

Assim, do ponto de vista prático, nosso estudo contribui com pelo menos duas ações que podem ser tomadas para que ambos os exemplos de dissonâncias de percepção sejam diminuídos. A primeira ação refere-se à organização de reuniões frequentes para alinhamento de metas, estratégias, bem como o estabelecimento de papéis definidos na equipe. Essas ações compreendem todas as variáveis do treinamento físico, técnico, tático e psicológico. Em nosso estudo, foi possível notar que as percepções do componente "treinamento físico" estão em concordância, o que não acontece, quando pensamos no componente psicológico (treinamento mental). Acreditamos que o alinhamento de informações na equipe pode facilitar a percepção e, consequentemente, as ações dos comportamentos envolvidos no processo de treinamento. 
A segunda proposta está diretamente relacionada com a primeira: possibilitar a presença do profissional da Psicologia do Esporte, uma vez que este tema permeia os conteúdos tanto da Psicologia do Esporte quanto da Educação Física com caráter interdisciplinar (VIEIRA; NASCIMENTO JUNIOR; VIEIRA, 2013). Assim, com diferentes profissionais integrados, o fenômeno estudado será melhor entendido, facilitando ações efetivas e claras, melhorando a comunicação entre todos os envolvidos no processo de treinamento esportivo: treinadores, atletas e outros profissionais. Somando aos argumentos anteriores, este estudo propõe que o olhar para a percepção do comportamento possa ser mais um passo na direção da compreensão do sujeito de forma global e integral (VIDUAL; FERNANDES, 2019), levando em consideração suas especificidades no processo de treinamento.

Neste estudo, houve boa interação do treinador com os atletas, reforçando que o treinador é um referencial na orientação, identificação e delimitação dos objetivos na modalidade esportiva (SILVEIRA, 2005). Além disso, a similaridade entre as respostas de ambos mostra que os treinadores buscam a melhoria nesses aspectos, incluindo-os na sua forma de lidar com os seus atletas e reforçando a ideia da comunicação ser um fator crucial para estimular, aumentar a autoestima e despertar o interesse do atleta pela modalidade (BRANDÃO; CARCHAN, 2010).

Por fim, considerando o tamanho amostral desse estudo, a generalização dos dados mostra-se limitada, porém a especificidade desse estudo por abordar um único esporte torna importante os resultados obtidos, reforçando a literatura sobre as outras modalidades mistas referentes ao comportamento do treinador na percepção dos atletas. Outro fator que pode ser levado em conta são as respostas adquiridas por meio dos questionários, uma vez que as respostas podem ter sido influenciadas pelo estado emocional do atleta naquele dia que preencheu o questionário, porém eles puderam responder de acordo com a prevalência dos comportamentos de seus treinadores durante os treinamentos.

\section{CONCLUSÃO}

Neste estudo, tanto os atletas quanto os treinadores enxergaram-se de maneira positiva, sobretudo no relacionamento interpessoal e em questões específicas da modalidade. Nossos resultados sugerem que os treinadores estão preocupados com a formação abrangente de seus atletas. Essa preocupação parte do estreitamento das relações entre eles, criando um trabalho humanizado e afetuoso.

Acreditamos que essa relação é determinada pela comunicação e é fundamental para que os atletas se aprimorem nas questões físicas, táticas, técnicas e psicológicas. Para finalizar, é importante ressaltar que a relação treinador-atleta positiva deve ser fortalecida pelo treinador, proporcionando evolução no desenvolvimento integral do atleta.

\section{REFERÊNCIAS}

ALBUQUERQUE MORAES, L. C. C.; MEDEIROS FILHO, E. S.; LÔBO, I. L. B.; SILIVEIRA, D. R. Escala do comportamento do treinador: versão treinador (ECT-T) e versão atleta (ECT-A): o que o treinador diz é confirmado pelos seus atletas? Revista Brasileira de Educação Física e Esporte, São Paulo, v.

\section{4, n. 1, p. 37-47, 2010}

ARDUA, C. M.; MÁRQUEZ, S. Relação entre estilos de liderança do treinador e rendimento na natação sincronizada. Fitness \& Performance Journal, Rio de Janeiro, v. 6, n. 6, p. 394-97, 2007

BERGER, B. G.; OWEN, D. R. Stress reduction and mood enhancement in four exercise modes: Swimming, body conditioning, hatha yoga, and fencing. Research Quarterly for Exercise and Sport, London, v. 59, n. 2, p. 148-59, 1988.

BRANDÃO, M. R. F.; CARCHAN, D. Comportamento preferido de liderança e sua influência no desempenho dos atletas. Motricidade, Riveira da Pena, v. 6, n. 1, p. 53-69, 2010.

CATITA, L. A relação treinador-atleta. A importância do treinador na motivação e continuidade da prática de jovens atletas em luta. Luta Magazine, Rio de Janeiro, v. 1, n. 1, p. 21-30, 2002.

CHASE, N. L.; SUI, X.; BLAIR, S. N. Comparison of the health aspects of swimming with other types of physical activity and sedentary lifestyle habits. International Journal of Aquatic Research and Education, Bowling Green, v. 2, n. 2, p. 7, 2008

CHELLADURAI, P. Leadership in sports. In: TENENBAUM, G.; EKLUND, R. C. (Org.) Handbook of Sport Psychology. New Jersey: John Wiley and Sons. 2007. p. 113-35.

CHELLADURAI, P.; SALEH, S. D. Dimensions of leader behavior in sports: development of a leadership scale. Journal of Sport and Exercise Psychology, Champaign, v. 2, n. 1, p. 34-45, 1980.

CHEUCZUK, F.; FERREIRA, L.; FLORES, P. P.; VIEIRA, L. F.; VIEIRA, J. L. L.; NASCIMENTO JUNIOR, J. R. A. D. Qualidade do relacionamento treinadoratleta e orientação às metas como preditores de desempenho esportivo. Psicologia: Teoria e Pesquisa, Brasília, v. 32, n. 2, p. 1-8, 2016

CID, L.; SILVA, C.; ALVES, J. Actividade física e bem-estar psicológico: Perfil dos participantes no programa de exercício e saúde de Rio Maior. Motricidade, Riveira da Pena, v. 3, n. 2, p. 47-55, 2007.

CÔTÉ, J.; YARDLEY, J.; HAY, J.; SEDGWICK, W.; BAKER, J. An exploratory examination of the coaching behavior scale for sport. Avante Research Note, Gloucester, v. 5, n. 2, p. 82-92, 1999.

CRUZ, A. B.; KIM, H.-D. Leadership preferences of adolescent players in sport: influence of coach gender. Journal of Sports Science \& Medicine, Bursa, v. 16, n. 2, p. 172, 2017.

CRUZ, J. F.; DIAS, C.; GOMES, R.; ALVES, A.; SÁ, S.; VIVEIROS, I.; ALMEIDA S.; PINTO, S. Um programa de formação para a eficácia dos treinadores da iniciação e formação desportiva. Análise Psicológica, Lisboa, v. 19, n. 1, p. 171-82, 2001.

FORTES, L. de S.; LIRA, H. A. A. D. S.; LIMA, R. C. R. D.; ALMEIDA, S. S.; FERREIRA, M. E. C. O treinamento mental gera efeito positivo na ansiedade competitiva de jovens nadadores? Revista Brasileira de Cineantropometria \& Desempenho Humano, Florianópolis, v. 18, n. 3, p. 353-61, 2016.

GOMES, R.; PAIVA, P. Liderança, compatibilidade treinador-atleta e satisfação no andebol: percepção de atletas novatos e experientes. PsicoUSF, Campinas, v. 15, n. 2, p. 235-48, 2010.

GOULD, D; CARSON, S. The relationship between perceived coaching behaviors and developmental benefits of high school sports participation. Hellenic Journal of Psychology, Thessaloniki, v. 7, n. 1, p. 298-314, 2010.

HORN, T. S.; BLOOM, P.; BERGLUND, K. M.; PACKARD, S. Relationship between collegiate athletes' psychological characteristics and their preferences for different types of coaching behavior. The Sport Psychologist, Champaign, v. 25, n. 2, p. 190-211, 2011.

HOSHINO, E. F.; SONOO, C. N.; VIEIRA, L. F. Perfil de liderança: uma análise no contexto esportivo de treinamento e competição. Journal of Physical Education, Maringá, v. 18, n. 1, p. 77-83, 2007.

LEITÃO, J. C. G. de C. A relação treinador-atleta: percepção dos comportamentos de liderança e de coesão em equipas de futebol. 1999 
25f. Tese (Doutorado em Educação Física) - Universidade de Coimbra, Coimbra, Portugal, 1999.

LESSA, L. F.; MORAES, E. W.; LIMA, S. F. Percepção dos alunos-atletas sobre o estilo de liderança dos professores-treinadores de Teresina - PI. Revista Brasileira de Futsal e Futebol, São Paulo, v. 5, n. 18, p. 1, 2013.

LÔBO, I. L. B.; ALBUQUERQUE MORAES, L. C. C.; NASCIMENTO, E. Processo de validação da escala de comportamento do treinador: versão atleta (ECT-A). Revista Brasileira de Educação Física e Esporte, São Paulo, v. 19, n. 3, p. 255-65, 2005.

MATIAS, T. S.; HINKEL, D.; GONÇALVES, E.; BERNARDO, V. M. Perfil de liderança de treinadores de triatlo do Brasil. Revista Portuguesa de Ciências do Desporto, Porto, n. 14, Suppl. 1, p. 1009-20, 2014

BRASIL. Ministério do Esporte. Diagnóstico Nacional do Esporte. Brasília, 2015. Disponível em: <http://arquivo.esporte.gov.br/diesporte/>. Acessado em: 15 de maio de 2019.

SAAVEDRA, J. M.; ESCALANTE, Y.; RODRÍGUEZ, F. A. La evolución de la natación. Lecturas: Educación Física y Deportes, Revista Digital, Buenos Aires, v. 7, p. 66, 2003. Disponível em: <https://www.efdeportes.com/ efd66/natacion.htm>. Acessado em: 15 de maio de 2019.

SERPA, S. Treinador e atleta: a relação sagrada. In: BECKER, B. (Org.). Psicologia aplicada ao treinador esportivo. Novo Hamburgo: Feevale, 2002. p.18-67.

SHERMAN, C. A.; FULLER, R.; SPEED, H. D. Gender comparisons of preferred coaching behaviors in Australian sports. Journal of Sport Behavior, Mobile, v. 23, n. 4, p. 389, 2000.

SILVEIRA, R. da D. Processo de adaptação e validação da escala de comportamento treinadores versão - treinadores (ECT-T). 2005. $128 \mathrm{f}$. Dissertação (Mestrado em Educação Física) - Universidade Federal de Minas Gerais, Belo Horizonte, 2005.

SIMÕES, A. C. Estudo do comportamento de liderança dos técnicos de handebol. 1987. 74f. Dissertação (Mestrado em Educação Física) Universidade de São Paulo, São Paulo, 1987.

SMITH, R. E.; SMOLL, F. L.; CUMMING, S. P. Effects of a motivational climate intervention for coaches on young athletes' sport performance anxiety. Journal of Sport and Exercise Psychology, Champaign, v. 29, n. 1, p. 39-59, 2007.

TAHARA, A. K.; SANTIAGO, D. R. P.; TAHARA, A. K. As atividades aquáticas associadas ao processo de bem-estar e qualidade de vida. Lecturas: Educación Física y Deportes, Revista Digital, Buenos Aires, v. 11, n. 103, p. 1-4, 2006. Disponível em: <https://www.efdeportes.com/efd103/ atividades-aquaticas.htm>. Acessado em: 10 de fevereiro de 2019.

TEIXEIRA, L. B. Exercícios aquáticos na manutenção e redução do peso na obesidade infantil. 2008. 43f. TCC (Graduação em Educação Física) Universidade Estadual de Campinas, Campinas, 2008.

VELLA, S. A.; OADES, L. G.; CROWE, T. P. The relationship between coach leadership, the coach-athlete relationship, team success, and the positive developmental experiences of adolescent soccer players. Physical Education and Sport Pedagogy, London, v. 18, n. 5, p. 549-61, 2013.

VIDUAL, M. B. P.; FERNANDES, P. T. Avaliação psicológica nos esportes. In: FERNANDES, P. T. (Org.). Interdisciplinaridade na psicologia do esporte. Curitiba: CRV, 2019. p. 350.

VIEIRA, L. F.; NASCIMENTO JUNIOR, J. R. A. do; VIEIRA, J. L. L. O estado da arte da pesquisa em Psicologia do Esporte no Brasil. Revista de Psicología del Deporte, Cerdanyola del Vallès, v. 22, n. 2, p. 501-7, 2013.

VILANI, L. H. P. Liderança situacional II e a relação treinador-atleta em diferentes categorias de base no tênis de mesa nacional. 2004. 173f. Dissertação (Mestrado em Educação Física) - Universidade Federal de Minas Gerais, Belo Horizonte, 2004.

ZHANG, J.; JENSEN, B. E.; MANN, B. L. Modification and revision of the leadership scale for sport. Journal of Sport Behavior, Alabama, v. 20, n. 1, p. 105, 1997.

\section{CONFLITO DE INTERESSE}

Os autores do estudo declaram não haver conflito de interesses.

\section{ORCID E E-MAIL DOS AUTORES}

Milena Schiezari Ru Barnabe ORCID: 0000-0001-7236-0679.

E-mail: milena_barnabe@hotmail.com

Hélio Mamoru Yoshida

ORCID: 0000-0002-1189-9192.

E-mail: heliomyoshida@gmail.com

João Guilherme Cren Chimazzo

ORCID: 0000-0002-0185-3262.

E-mail: chiminazzo@hotmail.com

Vinicius Nagy Soares

ORCID: 0000-0003-0363-5186.

E-mail: viniciusnagy@gmail.com

Paula Teixeira Fernandes (Autora Correspondente)

ORCID: 0000-0002-0492-1670.

E-mail: paula@fef.unicamp.br 